\title{
Mobile Big Data in the fight against COVID-19
}

\author{
Richard Benjamins ${ }^{1, *}$, Jeanine $\operatorname{Vos}^{2}$ and Stefaan Verhulst ${ }^{3}$ \\ ${ }^{1}$ Telefónica, Madrid, Spain \\ ${ }^{2}$ GSMA, London, United Kingdom \\ ${ }^{3}$ GovLab, New York University, New York, New York, USA \\ *Corresponding author. E-mail: richard.benjamins@telefonica.com
}

Received: 14 December 2021; Accepted: 14 December 2021

\begin{abstract}
In this editorial, Guest Editors Richard Benjamins (Telefónica), Jeanine Vos (GSMA), and Stefaan Verhulst (Data \& Policy Editor-in-Chief) draw insights from a set of peer-reviewed, open access articles in a Data \& Policy special collection dedicated to the use of Telco Big Data Analytics for COVID-19.
\end{abstract}

\section{Policy Significance Statement}

The Telco Big Data Analytics for COVID-19 collection is collection of articles that showcase experiences from around the globe of telco-government collaborations, with the aim of learning from the collective COVID-19 experience and to deeply understand how telco mobile big data analytics can help prepare the world for future pandemics.

\section{Introduction}

Almost 2 years into the COVID-19 pandemic, parts of the world feel like they may slowly be getting back to (a new) normal. Nevertheless, we know that the damage is still unfolding, and that many countries around the globe remain in a state of crisis. Given the global nature of this disease and the potential for mutant versions to develop and spread, a crisis anywhere is cause for concern everywhere. The world remains very much in the grip of this public health crisis.

From the beginning, there has been hope that data and technology could offer solutions to help inform governments' response strategy and decision-making. This includes mobile big data analytics, whereby mobile network operators create mobility insights, dashboards, and other decision-making tools, generated from anonymized and aggregated telco data. This hoped-for capability results from a growing group of mobile network operators investing in systems and capabilities to develop such products and services for public and private sector customers. Their value has been demonstrated in addressing different global challenges, ranging from the possibilities offered by models to better understand the spread of Zika in Brazil to interactive dashboards that aid emergency services during earthquakes and floods in Japan. Yet, despite these experiences, many governments across the world still have limited awareness, capabilities, budgets, and resources to leverage such tools in their efforts to limit the spread of COVID-19 using nonpharmaceutical interventions (NPI).

This special collection of papers we launched in Data \& Policy examines both the potential of mobile data analytics, as well as the challenges faced in delivering these tools to inform government 
decision-making. Consisting of 14 papers from 71 researchers and experts from academia, industry, and government, the articles cover a wide range of geographies (21), including Argentina, Austria, Belgium, Brazil, Burkina Faso, Democratic Republic of Congo, Ecuador, Estonia, Europe (as a whole), France, Gambia, Germany, Ghana, Italy, Malawi, Morocco, Nigeria, Nordics, Sierra Leone, South Africa, and Spain (see Figure 1). Responding to our call for case studies to illustrate the opportunities (and challenges) offered by mobile big data in the fight against COVID-19, the authors of these papers describe a number of examples of how mobile and mobile-related data have been used to address the medical, economic, sociocultural, and political aspects of the pandemic.

\section{The Papers}

Focusing on the use of mobility data in Latin America and the EU, De Alarcon et al. (2021) include an overview of Telefonica's efforts to help policymakers determine which COVID-19 policies to implement, and for how long. While they report successes using "mobility indicators with high spatial granularity," they also detail many challenges and obstacles. What clearly stands out is the broad variety of different collaboration models between mobile operators and governments.

Focusing on the case of Ghana, Li et al. (2021) argue that many - although by no means all — societal questions can be answered through "a robust public-private partnership framework, a privacy-preserving technical setup, and a communications strategy." The authors acknowledge that such a framework is no panacea, but their suggestions offer a useful starting point for policymakers and researchers seeking to optimize the public good impact of telco data.

While many of the authors focus on the medical aspects of the pandemic, Coudin et al. (2021) take a somewhat different approach, showing in their analysis how network signaling indicators from three leading French mobile network operators were used to analyze and respond to the economic fallout of COVID-19 and the government's responses. Likewise, Arai et al. (2021) analyze how call detail records in the Gambia helped inform economic policymaking, as well as other aspects of the response to the pandemic.

Vespe et al. (2021) describe a thus far unique experience of a public-private partnership in which 17 mobile network operators (MNOs) shared anonymized and aggregated data with the European Commission's Joint Research Centre (JRC) to cover 22 EU member states in the fight against COVID19. Their research has led to the development of two tools to fight COVID-19 that are used by health authorities from several European countries.

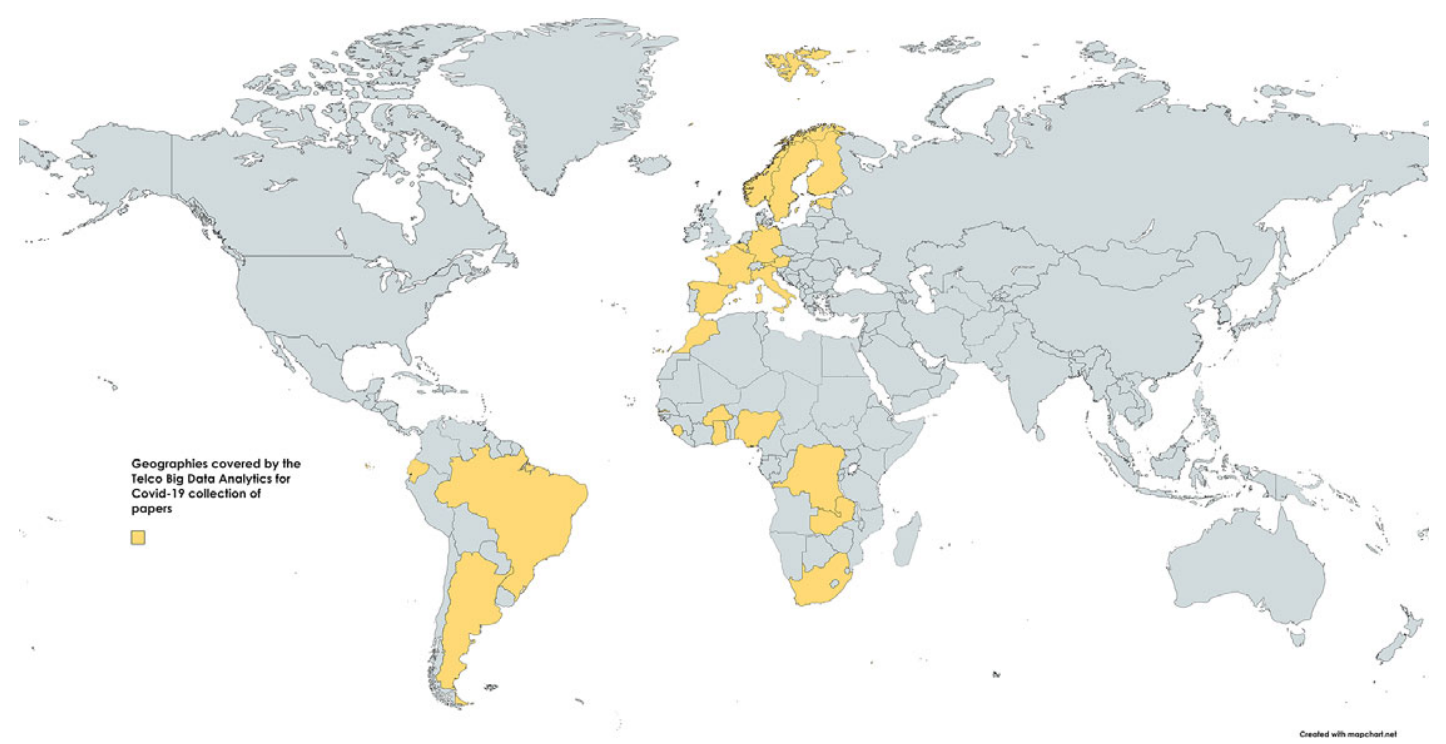

Figure 1. Geographies covered by the 14 papers. 
Gilbert et al. (2021) describe how MTN in Nigeria collaborated with the government to help plan the response to COVID-19. First, a model was developed to predict infections in each state, in order to support local resource planning decisions. And second, the parties worked to identify the most vulnerable people affected by the crisis, which helped inform financial relief efforts. Lessons learned include the important role of collaboration with and support from the regulatory authority and other stakeholders to deliver actionable insights at scale.

Ågren et al. (2021) explain the situation in the Nordic countries of Europe, and how Telia, a Swedish telecommunications company, has worked together with public health agencies to fight the epidemic. This paper shows that it is possible to form a mutually beneficial collaboration between a telecom operator and a public institution, and to make use of mobility data in evidence-based policymaking without compromising applicable personal data protection law.

Milusheva et al. (2021) note the proven efficacy of statistics derived from aggregated, anonymized mobile phone data as a proxy for mobility. They focus on drawing lessons learned and providing recommendations to unlock systematic use, based on their experiences of implementing collaborative projects around the world. Including raising more awareness, building capacity and skills, recognizing ongoing investment and funding requirements, undertaking appropriate privacy impact assessments, and focusing on longer-term deployments at scale.

Green et al. (2021) provide a case study of Malawi, where mobility dashboards were created to inform the Ministry of Health and Emergency Response Centre in their strategies and resource allocations during the pandemic. The paper describes how these dashboards helped identify possible chains of spread, irregular high-volume movements, and physical interconnectedness between regions. The dashboards provided vital information for government agencies to support decision-making, and the authors suggest the further applicability of such tools for other disasters; natural and human-made.

Snel (2022) shares lessons learnt from the DRC, where the Congolese public health authorities, through a steep learning curve, have initiated a public-private sector dialogue with local MNOs and their ecosystem partners to leverage population mobility insights for COVID-19 policy-making.

Jansen et al. (2021) focus on a vital aspect of using mobile operator data in policy, namely how to ensure and maintain public trust. The paper outlines five core governance principles that can act as a checklist for implementing organizations: necessity and proportionality, professional independence, privacy protection, commitment to quality, and international comparability. The authors bring these principles to life through discussions of specific projects in Estonia, the Gambia, and Ghana.

Calabrese et al. (2021) present the work conducted by Vodafone to enrich the understanding of people movement in Italy during the outbreak of the Coronavirus in 2020, and the tool developed to support the decisions taken by the authorities during that period. Vodafone have developed a solution to anonymously monitor the daily movements of Vodafone SIMs in Italy, at aggregate level, at different spatial and temporal granularity, to provide insights into the movements of Italians. Lourenco et al. (2021) reflect on Vodafone's global response to the pandemic and its collaboration with national governments and supranational entities, analyzing the methodologies that were developed and the regularity challenges found.

Chambreuil (2022) at Orange demonstrates how mobile positioning data (MPD) has been used to help combat COVID-19 in Europe, the Middle East, and Africa, and argue that MPD and data analysis can serve powering economic development as well as working toward the Sustainable Development Goals, while respecting data privacy.

\section{Analysis}

The call for these papers was originally made in the Summer of 2020. Fourteen contributions from all over the world were invited for submission. An initial set of five papers was published in June to coincide with the GSMA Mobile World Congress Barcelona. The next six papers were published to coincide with the UN World Data Forum (2-5 October), and the last three papers are now also published.

Although the world was already in the throes of the pandemic at the time of our original call, in retrospect, it is clear that we understood very little. Despite recent glimmers of hope, the overall story since 
that time has not been particularly illuminating: new variants of the virus continue to arise, creating uncertainties about the continued effectiveness of vaccines, and more generally of the future trajectories of societies and economies.

Nonetheless, an analysis of the papers leads to certain conclusions, which we summarize below.

Perhaps most importantly, it is essential to recognize that technology is not a silver bullet. Overcoming this ongoing crisis will require a multipronged approach that takes into account health and economic policymaking, awareness raising, and, not least, human behavior. Still, technology undoubtedly offers one avenue for progress, and the papers in this special collection show us a way.

In general, we have found it useful to analyze the papers along four key axes, each of which provides insights on a particular aspect of using telco data to fight pandemics and contribute to the public good. These axes include governance, capacity building, financial sustainability, and privacy.

A governance framework defines the rules of collaboration between public bodies, the private sector, and other types of institutions for data sharing. Ideally such a framework is comprehensive, defining all important aspects. Indeed, the business-to-government data sharing expert group of the European Commission, in its final report, came up with such an encompassing governance model. One consistent finding of these papers, however, is that the current data sharing economy lacks such an encompassing framework. Below, we categorize the different government actors as users of the data (Figure 2); as well as who has initiated the collaboration: government, private sector, or NGO (Figure 3).

Capacity building is related to the degree to which stakeholders are able to execute projects. Stakeholders include the private sector as data analytics and insights providers; governments that need to act based on the insights; and a variety of other stakeholders, including intermediate facilitating companies, sectoral associations, or humanitarian organizations (see Figure 4 for types of stakeholders involved as facilitating or intermediate organizations: universities, national statistics offices (NSO), thirdparty companies, NGOs, or industry associations). The papers show that most stakeholders face execution challenges for a variety of reasons, and that governments, in general, face the most challenges in making data-driven decisions or implementing evidence-based policymaking and monitoring.

Financial sustainability refers to the extent to which an initiative can continue over time in a sustainable way. Assessing sustainability requires asking a number of questions. Who is paying for the

\section{Government user}

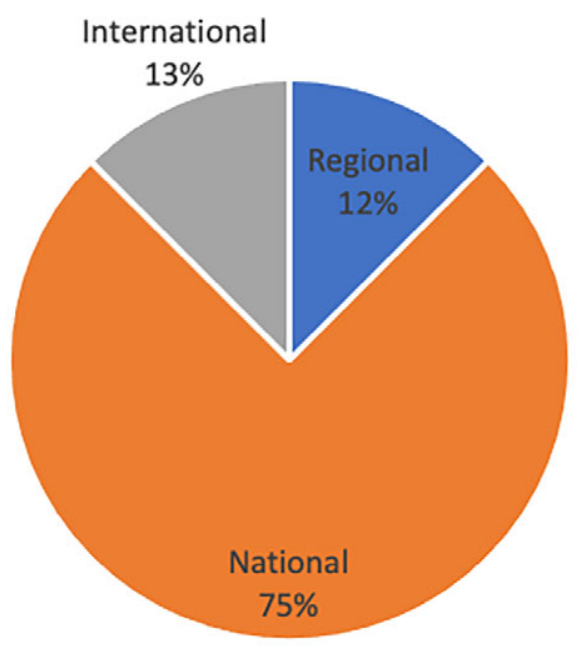

Figure 2. Type of government as final user. 


\section{Initiator of collaboration}

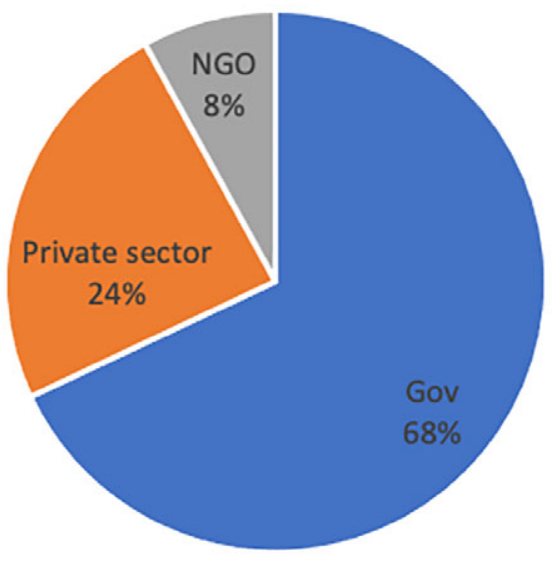

Figure 3. What organization starts sharing.

\section{Intermediate organization}

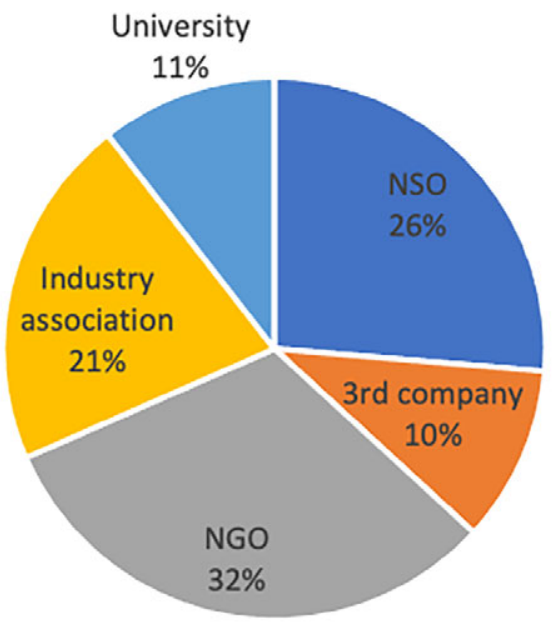

Figure 4. Percentage of organization type facilitating the data sharing.

costs needed to set up and operate a system? Is it an initiative pro-bono, or is there a commercial aspect? Are commercial terms clear and guided by a contract? In most of the initiatives under review, no commercial contract was in place, and almost all took place on a pro-bono basis (Figure 5).

The last dimension we analyzed in the initiatives of the 21 geographies is how issues surrounding privacy were handled. In some cases, described in the papers, privacy is ensured by the direct data holders/ 


\section{Type of contract \& funding}

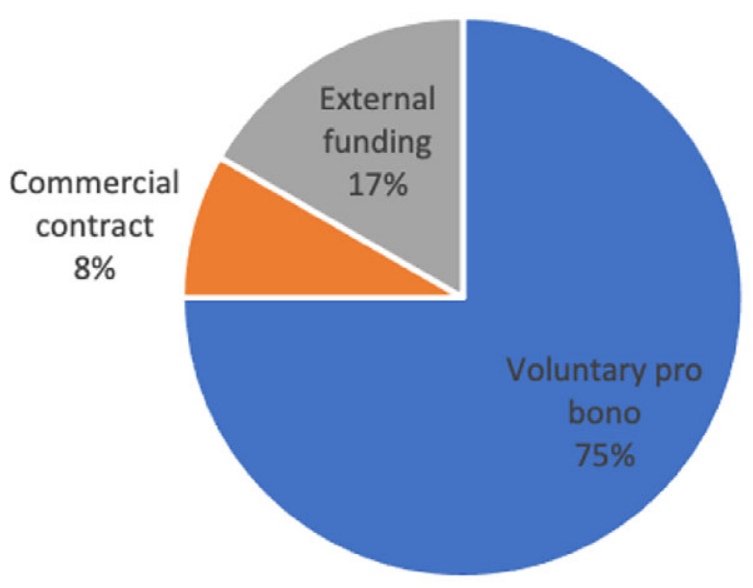

Figure 5. Type of agreement with relevance for financial sustainability.

providers involved, while in others, regulator or data protection agencies are involved. Although we found no consistent pattern, in general, all initiatives placed data responsibility at the core of their undertakings, and implemented human and technological methods to ensure that relevant privacy regulations and laws were complied with.

If there is an overarching theme that emerges from these papers, it is two-pronged. First, we learn that the potential of mobile data analytics to inform policy responses in pandemics is real; second, we equally learn that the current government, regulatory and business ecology to build and sustain that potential remains undeveloped. As Li et al. argue, even once a framework for collaboration between the private and public sectors is in place, "the challenges of competing priorities, managing expectations, and maintaining communication with data consumers during a pandemic mean that the potential of the data is not automatically translated into direct impact."

The authors of all these papers point to various specific failings and lacunae, and in doing so, they implicitly and explicitly suggest remedies. These suggestions provide avenues for further research. In this sense, we consider this collection a first step in an ongoing process, and we look forward to publishing further research. We look forward to reader feedback on the case studies offered here-and on other case studies or aspects that illuminate how telco data can be enlisted in the fight against COVID-19.

\section{How to Move Forward}

This collection demonstrates the positive contribution of telco big data analytics in creating powerful insights and tools to inform governments' response to the COVID-19 pandemic. Several papers further indicate how these solutions could also be beneficial in helping achieve other health, environmental, and societal goals. Nevertheless, important challenges still need to be overcome to realize systemic, at-scale deployments around the world. A number of recommendations emerge.

It is vital to continue raising awareness on the role that telco big data analytics can play, and moreover, to build capacity and digital skills within government agencies. Building capacity includes practical education and guidance, for example, creating better understanding on how to formulate needs and 
requirements, how to safeguard privacy and adopt ethical principles, and how to embed these tools into government decision-making processes.

Further, realizing appropriate funding is paramount to ensure the long-term systemic supply of telco big data insights and tools. Given the urgency and scale of the pandemic, many organizations offered probono products and insights on short notice. However, this is not sustainable for ongoing services. To move from one-off collaborations to longer-term adoption of telco big data analytics products and services, it is vital to ensure sustainable financing. Financing is required, among other goals, to move from best effort to excellence, to cover the required investment in platforms and systems, drive innovation in processing, analytics and packaging, and to cover operational costs incurred by industry.

Finally, the papers also formulate a call to action, to build on these case studies and observations, and to replicate approaches and solutions beyond the immediate response to COVID-19. Telco big data analytics insights and tools can continue to aid governments in the critical next phase of societal and economic recovery, as well as beyond to support governments in tackling the world's challenges. This special collection is only a starting point, from which we hope a wealth of solutions can continue to evolve and global goals realized. As researchers, practitioners and responsible citizens, it is our aim and hope that the experiences reported in this special collection will constitute a learning platform, leading to the creation of a world-wide NPI and pandemics early warning and management system. We trust that, like today, there is a world-wide Tsunami alert system that was created after the 2004 disaster that killed more than 200,000 people, in several years, there will be a world-wide NPI pandemics early alert system. Governments should embrace the science and technology coming from academia and the private sector, and make the necessary investments for a thriving public-private partnership.

\section{Innovation in the Journal}

From a publishing point of view, these papers introduce two new innovations for Data \& Policy. First, they mark the introduction of a new category, Translational Papers: articles that are rigorous and peerreviewed, but focus on the use of data science principles, techniques, and technologies in downstream settings to improve policy outcomes. Second, with the consent of everyone involved, we elected to run these articles through an open peer review process - reviewer identities disclosed to authors and reviews published alongside the articles - in order to provide some transparency on the decisionmaking process. The "peer reviews" tab on each article includes the reviews and editor recommendation comments.

Funding Statement. This editorial received no specific grant from any funding agency, commercial or not-for-profit sectors.

Competing Interests. Richard Benjamins is employed by Telefónica, a Spanish telecommunications company. An article by authors at Telefónica was included in the special collection and was accepted for publication after an independent peer review process. Jeanine Vos is employed by GSMA, an industry organization that represents the interests of mobile network operators worldwide. Two GSMA articles were included in the special collection and that were accepted for publication after an independent peer review process.

Author Contributions. Writing - review \& editing: R.B, J.V., and S.V. All authors approved the final draft.

Data Availability Statement. Data availability is not applicable to this article as no new data were created or analyzed.

\section{References}

Ågren K, Bjelkmar P and Allison E (2021) The use of anonymized and aggregated telecom mobility data by a public health agency during the COVID-19 pandemic: learnings from both the operator and agency perspective. Data \& Policy, 3, E17. https://doi.org/ 10.1017/dap.2021.11

Arai A, Knippenberg E, Meyer M and Witayangkurn A (2021). The hidden potential of call detail records in the Gambia. Data \& Policy, 3, E9. https://doi.org/10.1017/dap.2021.7 
Calabrese F, Cobelli E, Ferraiuolo V, Misseri G, Pinelli F and Rodriguez D (2021) Using Vodafone mobile phone network data to provide insights into citizens mobility in Italy during the Coronavirus outbreak. Data \& Policy, 3, E22. https://doi.org/10.1017/ dap. 2021.18

Chambreuil (2022) The value of network data confirmed by the Covid-19 epidemic and its expanded usages. Data \& Policy, 4. https://doi.org/10.1017/dap.2021.31

Coudin E, Poulhes M and Suarez Castillo M (2021) The French official statistics strategy: combining signaling data from various mobile network operators for documenting COVID-19 crisis effects on population movements and economic outlook. Data \& Policy, 3, E10. https://doi.org/10.1017/dap.2021.1

De Alarcon P, Salevsky A, Gheti-Kao D, Rosalen W, Duarte M, Cuervo C, Muñoz JJ, Pascual JM, Schurig M, Treß T, Diaz E, de la Cuesta C and Frias-Martinez E (2021) The contribution of telco data to fight the COVID-19 pandemic: experience of Telefonica throughout its footprint. Data \& Policy, 3, E7. https://doi.org/10.1017/dap.2021.6

Gilbert J, Adekanmbi $\mathbf{O}$ and Harrison C (2021) Using mobile big data to support emergency preparedness and address economically vulnerable communities during the COVID-19 pandemic in Nigeria. Data \& Policy, 3, E21. https://doi.org/ 10.1017/dap.2021.12

Green D, Moszczynski M, Asbah S, Morgan C, Klyn B, Foutry G, Ndira S, Selman N, Monawe M, Likaka A, Sibande R and Smith T (2021) Using mobile phone data for epidemic response in low resource settings — a case study of COVID-19 in Malawi. Data \& Policy, 3, E19. https://doi.org/10.1017/dap.2021.14

Jansen R, Kovacs K, Esko S, Saluveer E, Sõstra K, Bengtsson L, Li T, Adewole WA, Nester J, Arai A and Magpantay E (2021) Guiding principles to maintain public trust in the use of mobile operator data for policy purposes. Data \& Policy, 3, E24. https://doi.org/10.1017/dap.2021.21

Li T, Bowers R, Seidu O, Akoto-Bamfo G, Bessah D, Owusu V and Smeets L (2021) Analysis of call detail records to inform the COVID-19 response in Ghana—opportunities and challenges. Data \& Policy, 3, E11. https://doi.org/10.1017/dap.2021.5

Lourenco P, Kaur G, Allison M and Evetts T (2021) Data sharing and collaborations with Telco data during the COVID-19 pandemic: a Vodafone case study. Data \& Policy, 3, E33. https://doi.org/10.1017/dap.2021.26

Milusheva S, Lewin A, Begazo Gomez T, Matekenya D and Reid K (2021) Challenges and opportunities in accessing mobile phone data for COVID-19 response in developing countries. Data \& Policy, 3, E20. https://doi.org/10.1017/dap.2021.10

Snel N (2022) Turning Mobile Big Data Insights into public health responses in times of pandemics: lessons learnt from the Democratic Republic of the Congo. Data \& Policy, 4, https://doi.org/10.1017/dap.2021.30

Vespe M, Iacus S, Santamaria C, Sermi F and Spyratos S (2021) On the use of data from multiple mobile network operators in Europe to fight COVID-19. Data \& Policy, 3, E8. https://doi.org/10.1017/dap.2021.9

Cite this article: Benjamins R, Vos J and Verhulst S (2022). Mobile Big Data in the fight against COVID-19. Data \& Policy, 4: e9. doi:10.1017/dap.2021.39 